\title{
Antoine Berman et la traduction transparente : Le rendez-vous manqué de deux approches contemporaines de la pensée traductive
}

Jonas Tophoven*

Tout au début de la première partie de la thèse de doctorat que Solange Arber vient de soutenir à Lausanne et à Paris sur le thème « Elmar Tophoven et la traduction transparente » sous la direction conjointe de Bernard Banoun (Sorbonne Université) et Irene Weber Henking (Université de Lausanne), le «Portrait du traducteur en auteur » de la première partie démarre sur une longue citation tirée de la dernière page, la fin du chapitre XII de l'Amyot d'Antoine Berman, consacrée à "L'après-Amyot». Après avoir forgé à l'occasion de cet ouvrage une nouvelle notion de la critique traductive, la copia, Antoine Berman l'y reformule comme une sorte de projet permettant au traducteur de « trouver sa positivité » : «La vocation du traducteur est la pluralité. Plus encore : le vrai traducteur est polyvalent au-delà de la traduction ». Selon Antoine Berman, son contemporain Elmar Tophoven fait partie de la liste de ceux qui possèdent déjà cette copia. Son nom est mentionné dans une phrase elliptique placée à la ligne, dessinant une figure de style, en chiasme, avec la précédente :

En France, Amyot, Robin, Larbaud (grand introducteur sinon grand traducteur) possédaient cette copia.

* Journaliste. 
Schlegel, George, Celan et Tophoven en Allemagne, aussi.

Le paragraphe précédent est tout aussi court et forme avec les deux suivants une sorte de triade :

A cette copia de l'activité traductive, joyeuse comme toute copia, le traducteur n'a guère encore accès.

Intercaler parfois des paragraphes courts qui sont en fait des sentences, cela fait partie du style d'Antoine Berman, tout comme l'usage des italiques, des parenthèses. Chez lui, les italiques ont une double fonction, technique et emphatique. La mise en italique du terme copia tient des deux. Il faut cependant remarquer que l'auteur utilise aussi les doubles guillemets. Si l'on prend l'exemple de ces toutes dernières pages de l'ouvrage, Antoine Berman écrit un peu avant : «En fait, plus qu'aider, la traduction doit être cultivée ». Alors que le paragraphe qui précède la triade s'achève sur un emprunt à Freud et Lacan, qui reprend le terme de culture en forme substantivée mais placée entre des doubles guillemets. Je pense que ces derniers soulignent l'emprunt, tandis que la mise en italiques est une accentuation.

Si l'on reprend cette triade, elle appelle plusieurs remarques :

- la première sentence se projette dans le futur avec une sorte de contradiction : si le traducteur n'a pas eu accès à la copia jusqu'ici, pourquoi, la phrase suivante, l'auteur dénombre-t-il des traducteurs qui y ont eu accès ? Il est vrai que la phrase négative comporte le mot «guère » qui évoque une notion de quantité, avec deux acceptions possibles : soit les traducteurs passés cités n'avaient qu'un peu de copia chacun, soit il s'agit de quelques rares exceptions. Or, Antoine Berman écrit que ces traducteurs, à la fois les Français et les Allemands, possèdent cette copia, sans restriction.

- le style de la triade est elliptique, une énumération de noms de famille, Schlegel n'est pas spécifié. Le contexte fait pencher pour A.W. Schlegel, le traducteur de Shakespeare. Un lecteur français est-il censé connaître Stefan George, Paul Celan, Elmar Tophoven ? La remarque 
précédente nous incite à être attentif à la lettre du texte. Il a été question de «Freud et Lacan ». A.W. Schlegel perd son prénom et initie une double série de noms de famille. $S^{\prime} y$ exprime une volonté stylistique qui confère à l'ensemble de la triade une accentuation.

- Valéry Larbaud est évoqué entre parenthèses comme "grand introducteur sinon grand traducteur». Que veut-il dire ? Y-a-t-il un jugement de qualité, Antoine Berman préfère-t-il l'auteur de «Sous l'invocation de Saint Jérôme » (une lecture de chevet d'Elmar Tophoven), au traducteur de Joyce ? Ou bien l'adjectif "grand» a-t-il une valeur quantitative?

- la sentence française évoque trois noms, la suivante quatre. Il y a un déséquilibre qui invite à lire qu'au-delà du parallélisme, après mûre réflexion, en se risquant un peu, on peut dire que Tophoven fait partie aussi du lot prestigieux.

- dans la formule "et Tophoven en Allemagne, aussi », le terme Allemagne ne désigne pas un lieu mais un espace culturel pendant $\mathrm{du}$ français. A vrai dire, ni Celan ni Tophoven ne sont en Allemagne et la formulation est plutôt expéditive et laconique pour ce qui concerne Paul Celan. Pourquoi Antoine Berman se focalise-t-il sur ces deux langues ? Pourquoi le fait-il en ajoutant une liste de noms propres de traducteurs allemands qui est un peu déroutante surtout en s'achevant sur Tophoven ? Ni George, ni Celan, ni Tophoven n'ont été évoqués dans l' Amyot. Il y a deux façons d'interpréter le geste d'Antoine Berman: une figure de style calculée, ou bien justement l'une de ces sentences lapidaires, visionnaires et même un peu prophétiques. De temps en temps, et notamment à la fin de ce long texte, comme de raison, l'auteur lève les yeux et regarde au loin. Il ne distingue pas des contemporains comparables à Amyot, sans doute à cause du carcan culturel qui sévit encore. Son regard revient en arrière, établit le lien entre son Amyot et L'épreuve via Schlegel, sa connaissance de l'histoire de la traduction le mène de Schlegel à George (et pas à Benjamin !), de George à Celan (malgré le hiatus politique), et de Celan il glisse à Tophoven, qu'il embarque malgré tout, pas grand poète, mais justement traducteur, figure qui cadre avec ce qu'il établit en relation avec une figure positive. Tandis que le concept même de copia est une sorte d'envol audacieux, un saut dans 
l'inconnu pour prendre le contrepied des notions ancrées et dominantes, l'énumération de George, Celan et Tophoven est pour le lecteur français teinte de mystère, tandis que pour lui, il y a là un autre cheminement audacieux et rapide de la pensée. Sa notion de copia risque de partir en fumée, au-delà d'Amyot, et de Robin, déjà, Larbaud ne cadre pas parfaitement. Il n'écrit pas Schlegel, Luther ou l'inverse. Il semble ouvrir une porte sur l'inconnu en même temps qu'il rend hommage à son ami disparu. Cette porte, c' est celle dont il est précisément question ici.

\section{La copia}

Dans l'Amyot, Antoine Berman centre un chapitre entier, le VIII, sur cette notion, mais, si l'on compare le travail conceptuel de cet ouvrage à celui du John Donne, on a l'impression que le travail conceptuel se focalise ici sur cette dernière. Quant au chapitre final consacré à l'après-Amyot, il donne quelques indications sur ce que cette notion peut vouloir dire quand elle est appliquée à des figures plus contemporaines. Antoine Berman, qui évoque les « fières déclarations de Luther, de A.W. Schlegel, ou de Robin » en ajoute à son tour en écrivant : «La vérité de la traduction est dans la plénitude de l'amour ». La phrase renvoie clairement à Valéry Larbaud dont le recueil «Sous l'invocation de Saint Jérôme » était bien connue d'Elmar Tophoven, sans pour autant qu'il reprenne à son compte cette métaphore amoureuse dans ses propres textes sur la traduction. Il va de soi que cette image de l'amour est dérivée de celle de l'infidélité dont le traducteur est affublé. Antoine Berman cherche à retourner l'image culturelle, lui formuler un inverse positif. Valéry Larbaud le précède par l'image de la séduction amoureuse, qu'Antoine Berman pousse encore un peu plus loin vers la pleine jouissance mutuelle, sans original sexué. L'Amyot et peut-être la pensée d'Antoine Berman sur la traduction culmine dans cette phrase qui invite à une compréhension intuitive. 


\section{In Memoriam}

L'hommage appuyé mais laconique de la fin de l'Amyot trouve son pendant dans le texte nécrologique rédigé par Antoine Berman à la suite du décès de son ami, assez court pour être repris ici dans son intégralité :

In memoriam Elmar Tophoven

Elmar Tophoven a d'abord été un grand traducteur : celui de Beckett, au premier chef, mais aussi celui du «nouveau roman » français, Nathalie Sarraute, Claude Simon, Robbe-Grillet... Il a également traduit des fragments de Rabelais - admirablement - pour la Compagnie RenaudBarrault. Cette incessante activité de traduction n'a cependant pas absorbé toutes ses énergies : il a fondé le "Collège européen des traducteurs » de Straelen, en Allemagne Fédérale, le premier de ces lieux de rencontre, d'étude et de travail pour traducteurs littéraires qui depuis, à son active instigation, se sont multipliés en Europe. Elmar Tophoven est enfin, et peutêtre surtout, le promoteur d'une manière nouvelle de pratiquer la traduction, à la fois plus rigoureuse, plus moderne et plus généreuse. Pas seulement parce qu'il a été le premier à percevoir les merveilleuses potentialités que l'informatique offrait aux traducteurs littéraires. Mais parce qu'il a forgé le concept - et la pratique - de ce qu'il appelait la «traduction transparente». D'une traduction qui, réfléchissant constamment sur elle-même, enregistrant ses étapes, ses phases, ses processus, garde mémoire d'elle-même, et peut, ainsi, à la fois promouvoir - pour la première fois sans doute dans l'Histoire - un échange fécond entre les traducteurs, une transmission d'expérience et, au-delà, permettre, pour la première fois également, un enseignement concret et systématique de la traduction littéraire. Au traducteur «traditionnel», solipsiste et intuitif, Tophoven oppose l'idéal (par lui déjà incarné) d'un traducteur " généreux », soucieux de transmettre son savoir, et systématique dans son agir. Ces idées ont eu un vaste écho dans le monde de la traduction, et elles vont peu à peu - telle est leur force agissante - le révolutionner.

Tout cela n'a pas été sans soulever maintes oppositions et maints conflits, mais l'esprit du travail de Tophoven a d'ores et déjà commencé à transformer et les méthodes et l'esprit même de la traduction littéraire.

En 1989, Antoine Berman a bien saisi la triple spécificité de celui qui, disons-le tout de suite, fut mon père : traducteur littéraire, fondateur du Collège européen de Straelen en Allemagne, laborantin d'une sorte de 
méthode ou d'approche pratique de la traduction littéraire qui fait précisément l'objet de la thèse de Solange Arber. On comprend tout de suite que pour Antoine Berman, c'est cette pluralité ou polyvalence au-delà de la traduction qui justifie la mention du nom de mon père aux côtés des figures illustres. Elmar Tophoven a la copia car il n'est pas resté dans le rôle culturel effacé et servile qu'on assigne habituellement à un traducteur. Son foisonnement lui rappelle précisément celui de Jacques Amyot, qu'il érige comme figure tutélaire de la traduction française, ou son homologue allemand Schlegel.

\section{Eléments biographiques}

Il n'est plus possible de retracer précisément comment Antoine Berman et Elmar Tophoven se sont connus. Tout commence au retour d'Antoine et d'Isabelle de leur séjour en Argentine, dans la seconde moitié des années 70. Erika Tophoven, qui a travaillé depuis une vingtaine d'années comme aide et relais de son mari traducteur, s'émancipe et choisit de traduire elle-même vers l'allemand des ouvrages (Charles-Roux, Simenon, Bille, Cixous, Segalen...). Accessoirement, elle se charge également de traductions commerciales, au même titre que son mari exerce parallèlement la profession de speaker, après avoir travaillé des décennies durant comme guide touristique. Erika Tophoven traduit notamment en allemand des articles de la revue Mon tricot du groupe Boussac, un travail bien payé et réputé stable jusqu'au déclin et démantèlement de cet empire. Isabelle Berman traduit également Mon tricot, vers l'espagnol. Il semble que c'est comme cela que le premier contact a été établi. A l'époque, la création du Collège européen des traducteurs de Straelen est déjà bien avancée, il existe une association, une maison provisoire est mise en service en 1980 dans l'attente de l'inauguration en 1985 du Collège sous la forme qu'il a encore aujourd'hui, par la réhabilitation et le regroupement d'un groupe de maisons parmi lesquelles se trouvent, par hasard, la maison de naissance d'Elmar. Les Tophoven habitent en lointaine banlieue parisienne, puis à St Germain-en-Laye, et ne reviennent s'établir à Paris qu'en 1980. 
Au début, sans doute, Elmar cherchait des émules pour sa méthode de traduction transparente. C'est ainsi qu'à partir de 1980, à l'occasion de la traduction de nombreuses œuvres de Theodor Fontane, qu'il anime pour la Fondation Robert Bosch, il tente en vain, mais en faisant beaucoup d'efforts, de propager, sachant que les traducteurs se voient mis à disposition des ordinateurs portables de la première génération. Or, à l'époque, Antoine pratique la traduction avec Isabelle mais sans établir des fiches pour noter ses trouvailles. Question de complexion ou contraintes économiques ? Antoine est au courant de la méthode Tophoven, mais il ne l'applique pas, lui non plus. Parallèlement, il est occupé à achever sa thèse sur la traduction à l'époque du romantisme allemand. Cette fois, c'est Elmar qui a du mal à suivre, ne fréquentant guère tous ces auteurs. Ce qui ne l'empêchera pas de lire avec intérêt la thèse d'Antoine quand elle sera publiée en 1985.

Les échanges sont plus simples à partir de 1980, à cause de la proximité géographique entre les deux domiciles parisiens. Mais Elmar est déjà très souvent à Straelen ou bien voyage dans le monde pour faire la promotion du Collège. Il est arrivé qu'Antoine passe rue St Jacques avec son bébé Nicolas dans une poussette, fier sans doute de le présenter, d'autant que je me souviens que nous avions suivi de près la période de la grossesse. Je me souviens que ma mère a assisté à l'une ou l'autre conférence d'Antoine à Paris, surprise par son assurance. Parallèlement au Collège de Straelen, un second Collège a vu le jour à Arles, porté par une association qui organisait chaque année ses Assises en grande pompe. Mes parents étaient impliqués, et Antoine était un intervenant demandé. Mais je me souviens que l'un des organisateurs s'est plaint auprès de moi de ce qu'Antoine soit un peu « l'Arlésienne ». J'en ai fait part à Antoine qui en a été un peu pincé, et m'a répondu qu'il se consacrait prioritairement à son Centre et à sa famille. De fait, Antoine était très occupé par ses cours au Collège de philosophie, également, où sa réflexion portait sur bien d'autres choses que les Collèges, dont il aurait pu devenir le parrain intellectuel.

Il existe tout de même un écart fondamental entre la démarche de l'un et de l'autre. Elmar imagine que le traducteur note ses trouvailles et difficultés pour que cela serve à la science linguistique. Plus précisément, le Collège est à la fois un lieu d'échange entre traducteurs et un lieu de transfert 
de ces traces de la pratique vers la science. Elmar ne cesse de chercher des moyens de justifier et d'étayer sa démarche et s'appuie alors notamment sur les remontrances d'Henri Meschonnic qui demande que le traducteur rende des comptes. En gros, la démarche d'Elmar est tout aussi disruptive dans la pratique que celle d'Antoine l'est sur le plan de la pensée. A l'époque, faire de la traduction un objet d'analyse suscite la même perplexité que lorsque Pastoureau s'intéresse aux couleurs. Cela vaut pour l'analyse pratique ou l'analyse globale menée par Antoine à une époque qui se poursuit jusqu'à aujourd'hui, où la légitimité d'un discours sur la traduction requiert une base épistémologique et scientifique. On retrouve d'ailleurs cet étai dans la méthode Tophoven qui tend la main à la linguistique, sans que, d'ailleurs, cette science soit capable de la saisir.

Deux sensibilités sont à l'œuvre sans s'opposer mais en poursuivant leur trajectoire de comète. Un jour, en 1985, il est question d'un discours que le roi d'Espagne doit tenir à Aix-la-Chapelle à l'occasion d'un prix qui lui est attribué. Erika Tophoven, l'épouse d'Elmar, qui se trouve à Straelen, suggère que ce serait l'occasion de lui faire mentionner le Collège comme nouvelle école de Tolède. L'idée fait son chemin et de fait, le roi d'Espagne mentionne dans son discours le Collège de Straelen. A la suite, il adresse à Elmar une lettre où il le qualifie de "cher ami ", ce qui le flatte. Elmar est alors déjà malade, il voudrait répondre à cette lettre et se rend chez les Berman où Antoine lui rédige la réponse tandis qu'Elmar se charge de pouponner (il s'agit alors d'Alexandre).

C'est d'ailleurs la période où, le Collège étant inauguré, Elmar tente d'organiser des séminaires qui sont financés mais aussi cadrés par les fondations comme Bertelsmann, et organisés par l'association syndicale des traducteurs. Dans les archives Tophoven de Straelen, on trouve le compterendu d'une réunion de l'été 1986, visant à planifier les séminaires proposés aux auteurs et aux traducteurs.

\section{Pensées croisées}

Comme le détaille Solange Arber, l'essentiel de la méthode de traduction d'Elmar Tophoven se met en place au cours des années 70. Dès la création 
du Collège provisoire à Straelen en 1978, Elmar Tophoven organise des séminaires de traduction $\mathrm{du}$ français vers l'allemand à destination de traducteurs littéraires professionnels, une pratique prolongée ensuite par le traducteur Joseph Winiger. C'est aussi l'époque où il accompagne le projet de traduction des œuvres de Theodor Fontane en français, projet parrainé par la fondation Bosch et qui donne lieu également à un séminaire à Straelen durant l'été 1979. Les années 80 devraient être la consécration de la démarche accomplie, grâce à la mise en service du Collège définitif comme lieu de pratique de la traduction et d'échange autour de cette discipline. Mais les choses ne se passent pas comme prévu. L'inauguration coïncide avec le diagnostic d'un cancer, l'écart entre les orientations préconisées par Klaus Birkenhauer et Elmar Tophoven ne cessent d'augmenter jusqu'à mener à un clash dont Klaus Birkenhauer sort vainqueur. Surtout, tous les efforts d'Elmar Tophoven n'ont pas abouti à ce que d'autres traducteurs adoptent, sinon son épouse, cette approche transparente, ne serait-ce que sous sa plus simple expression, qui est de noter les étapes de recherche de solutions menant à des trouvailles qui sont ensuite réunies dans un glossaire, et partagées.

Dans son texte nécrologique, Antoine Berman fait allusion aux difficultés rencontrées, qui obscurciront les dernières années de maladie d'Elmar Tophoven. Mais il y voit l'expression inévitable des affres d'une profession inexorablement entraînée dans une révolution qui suit son cours. De cela, les décennies passées n'ont pas porté la trace, et si la vision d'Antoine Berman est prophétique, elle doit alors porter sur le temps long. L'ordinateur personnel n'a pas été utilisé par les traducteurs pour noter leurs trouvailles, mais pour traduire plus commodément. Très vite, le gain technologique a été absorbé et les tarifs sont revenus à leur bas niveau de fait. Au Collège, la mise en réseau alors unique n'a pas non plus été opérante, et tout le système informatique s'est écroulé un beau jour subitement à peu près au moment de la chute du Mur.

Alors que les années 80 sont pour Elmar Tophoven la décennie de l'échec, pour Antoine Berman, c'est la période de sa trop brève envolée. Mais il est bien trop accaparé par ses cours au Collège de philosophie et le développement de son Centre Amyot pour se consacrer au développement 
du Collège des traducteurs d'Arles, au grand regret de l'association d'ailleurs. Seul le projet de traduction de L'épreuve engagé par Elmar Tophoven l'été 1986 pour une session en février 1987 parviendra à attirer Antoine Berman pour quelques jours à Straelen.

On trouve cependant dans les archives familiales un tiré à part de l'article La traduction et ses discours qui remonte à la même époque (« La traduction et ses discours », in Confrontation, no. 16, automne 1986, p. 83-95), sans doute consulté au moment de la préparation du séminaire et annoté, du moins sur les cinq premières pages. Le tiré à part assez usé semble avoir beaucoup servi au demeurant. Difficile de dériver des soulignements une lecture proprement dite. Elmar Tophoven utilise son crayon comme outil de lecture et de compréhension tout en relevant les éléments qui se rapportent le mieux à ses préoccupations. L'article, repris en 1989 dans Meta, a son importance dans la mesure où Antoine Berman y définit la traductologie et ses tâches. Mais il n'est peut-être pas anodin que l'on ne trouve pas de soulignements dans cette partie centrale, comme si le sujet n'était pas vraiment celui d'Elmar Tophoven.

Peut-être qu'Elmar Tophoven n'aura pas remarqué la dernière note de l'article, qui se rapporte à la dixième tâche de la traductologie, la paideia traductive :

\footnotetext{
Que notre époque s'achemine vers une telle paideia, c'est ce qu'attestent les créations du Collège Européen des Traducteurs de Straelen (Allemagne Fédérale), du Collège International des Traducteurs d'Arles et, un peu partout, de nombreux « centres de recherches » sur la traduction. La traductologie n'est rien d'autre que la réflexion qui vient soutenir et éclairer (tout en s'y développant et approfondissant) l'activité de ces centres et collèges.
}

Si Elmar Tophoven n'a pas pu suivre le déploiement de la pensée d'Antoine Berman, à l'inverse, cette note montre qu'Antoine Berman semblait vouloir aller tout à fait dans le sens imaginé par le traducteur allemand et fondateur du Collège de Straelen, qui cherchait précisément à y établir un lieu de brassage entre la pratique et la théorie. 


\section{A l'épreuve de L'épreuve}

La première édition de L'épreuve de l'étranger paraît chez Gallimard en 1984. L'exemplaire des archives familiales est aussi usé et annoté que les autres originaux traduits par mon père. On y voit la tentative de procéder à une traduction transparente de ce texte. Ainsi, des soulignements de couleur numérotés renvoient à des fiches de traduction. Pour la préface intitulée "La traduction au manifeste", pas moins de 284 problèmes de traduction sont numérotés dans la marge. Dans les archives, on trouve effectivement 70 fiches pour les trois premières pages, rédigées de la main d'Elmar Tophoven, mais sans le tracé des étapes de recherche et sans classification par code couleur indiquant si le problème en question est plutôt lexical, syntaxique ou rythmique. Les fiches s'abstiennent de commenter le résultat, d'en tirer une leçon. Les fiches sont moins nombreuses pour le chapitre consacré à Herder puisqu'on ne trouve que 16 fiches. S'ajoutent deux fiches se rapportant à des segments soulignés au crayon à la page 76 dans le chapitre sur la Bildung. Le travail est inachevé. Et il applique la méthode à une situation de traduction en groupe. Les chapitres traduits par Elmar Tophoven sont, comme ceux traduits par les autres participants, des ébauches. Les fiches, qui sont d'ailleurs à cette époque le plus souvent produites à la machine, n'ont été confectionnées qu'après des séances qui valident la traduction. Elmar Tophoven établit ainsi un glossaire, malheureusement, il est seul à le faire.

Etonnamment, la traduction est manuscrite elle aussi, tout comme le sont les fiches cartonnées, comme au début des années 70. Sans doute, ce retour à la traduction manuscrite, relevé également par Solange Arber, s'explique par l'itinérance d'Elmar à cette époque.

Les participants à ce séminaire sont au moins en partie des habitués. Il s'agit du cinquième et dernier séminaire de formation continue pour traducteurs traduisants du français vers l'allemand. Tout comme des séminaires de formation continue d'auteurs également organisés au Collège mais n'ayant en principe rien à voir avec son essence, ces séminaires font l'objet d'un financement extérieur, tandis que le syndicat allemand des traducteurs fait office de secrétariat. Les séminaires précédents avaient été des succès, notamment celui dédié à Jules Renard. A noter qu'Elmar 
Tophoven a revu (avec moi) jusqu'à ses derniers jours sa traduction des Histoires naturelles jusqu'à ce que les doses de morphine deviennent trop fortes. Je n'ai d'ailleurs jamais eu le courage de remettre le nez dans un travail auquel j'ai sans doute pris une part indue.

Les quatre séminaires précédents n'ont pas pu susciter chez les participants une pratique de la traduction transparente. En amont, les participants doivent traduire des textes qui seront analysés au cours du séminaire. Ils s'acquittent de ce pensum imposé mais s'excusent de ne pas livrer de commentaire ou de trouvailles fichées. Dans le cas de L'épreuve, ils butent surtout sur le problème de la recherche des citations allemandes du texte.

Chaque participant a reçu par courrier un exemplaire de l'ouvrage d'ailleurs paru en 1985 en collection TEL. Ils livrent leurs traductions en sortie impression par courrier. Le séminaire est animé conjointement, sans doute pour décharger Elmar Tophoven, avec le traducteur Thomas Dobberkau.

Elmar Tophoven est assez familier du sujet, dans la mesure où l'ouvrage Weltliteratur, épais de 700 pages, qui accompagne en 1982 une exposition organisée par les archives littéraires de Marbach, est littéralement son livre de chevet. Le catalogue illustre "l'envie de traduire au siècle de Goethe ». Parallèlement à ses nombreuses lectures linguistiques, Elmar Tophoven est sensible à tout ce qui touche l'histoire de la traduction. Le choix de traduire L'épreuve dans ce séminaire se fait clairement dans la perspective de promouvoir l'ouvrage dans la perspective d'une publication en allemand. Elmar Tophoven procède déjà dans d'autres cas à ce type de travail en éclaireur. Il imagine, et n'a pas tort, que les éditeurs allemands pourront au moins se faire une opinion assez précise du texte, même s'il faudra engager tout un travail de lissage.

Elmar Tophoven poursuit donc plusieurs objectifs : sensibiliser ses confrères à la méthode de la traduction transparente, mener à bien ces séminaires dans leur continuité et comme possibilité de rencontre et d'échange au sein du Collège, et enfin promouvoir l'œuvre d'Antoine Berman. Il est un peu douloureux de constater que ces efforts sont mis en échec sur les trois plans. C'est notamment dommage pour la réception de 
l'œuvre d'Antoine Berman en Allemagne, qui est partie d'un mauvais pied et ne s'en est pas remise jusqu'à aujourd'hui.

Que s'est-il passé ? Elmar Tophoven a été de plus en plus confronté à sa maladie. Face à lui, il semble que ses détracteurs ont fait valoir que l'ouvrage d'Antoine Berman ne valait pas la peine d'être traduit car il s'agissait simplement d'une thèse. Toujours est-il qu'Antoine s'est vu notifier le refus des éditions Suhrkamp sur son lit de mort à l'automne 1991, quatre ans plus tard.

\section{Et depuis?}

Que reproche-t-on à la pensée d'Antoine ? En général, la réception se concentre sur L'épreuve de l'étranger, où le sous-titre, qui est une discipline imposée par les éditeurs, mentionne les Romantiques allemands alors que la thèse remonte jusqu'à Luther. Par ailleurs, comme le relève Dirk Weissmann en 2014, la thèse centrale d'Antoine Berman tient beaucoup à Schleiermacher sur le plan de l'articulation, et à Hölderlin sur le plan de la pratique. Dirk Weissmann constate qu'Antoine Berman a tiré Schleiermacher de son contexte en faisant de lui le chantre de la traduction comme épreuve de l'étranger alors qu'en fait, il aimerait bien voir l'Allemagne quitter enfin ce stade. Il est dommage que l'article de Dirk Weissmann ne tienne pas compte de l'Amyot paru en 2012, car la pensée d'Antoine y évolue vers l'idée d'un âge des traductions qui s'est également manifesté en France. Il manque donc en Allemagne une réception d'Antoine Berman qui comparerait l'âge de la traduction allemande (vers 1750-1850) et l'âge de la traduction française (13e siècle-16 ${ }^{\mathrm{e}}$ siècle). Par ailleurs, il serait intéressant de comparer, pour la fin du $20^{\text {e }}$ siècle, une langue française de décolonisation avec une langue allemande colonisée. C'est peut-être aussi cette différence fondamentale qui explique pourquoi la pensée d'Antoine Berman n'est pas reçue dans les pays germaniques, alors qu'elle y puise sa matière.

La tentative la plus importante pour favoriser la réception de l'œuvre d'Antoine Berman en Allemagne est à chercher dans la publication en Allemagne, et en allemand, de ce qui ressemble à une thèse. Irène Kuhn : «Antoine Berman : Das Projekt einer "produktiven" Übersetzungskritik», 
chez Günter Narr qui est entre autres un éditeur de thèses. Il semble bien qu'il s'agisse d'une thèse d'Etat française non soutenue car n'ayant plus cours, rédigée en allemand par l'enseignante française d'origine alsacienne. Paru en 2007 et augmenté de la traduction en allemand de «Pour une critique des traductions », le titre fait référence à la première partie du John Donne, qui est d'ailleurs traduite. Le travail a été commenté par le traducteur Rolf Pütter en 2010.

Cette incursion de la pensée d'Antoine Berman dans le monde allemand a laissé quelques traces mais sans inverser la tendance précédente. Ainsi, comme cela est le cas pour d'autres pays, la recherche sur Antoine Berman sur Google affiche à droite, quand des pages existent, une indication selon wikipedia, mais l'image associée est celle de Volker Braun, à cause d'un blog italien utilisant cette image près d'une citation traduite en italien d'Antoine Berman. Si la pensée d'Antoine Berman avait dans le monde germanique un véritable relai intellectuel, ce choses-là ne dureraient pas longtemps.

\section{Erreur de stratégie}

La préparation du séminaire, qui conduit Elmar Tophoven à traduire plusieurs chapitres de l'ouvrage, est aussi pour lui une façon de se plonger dans sa compréhension, de compléter sa culture de l'histoire de la traduction. Bien que la pensée d'Antoine Berman ne se résume pas à L'épreuve, loin de là, c'est à peu près l'horizon de sa perception par Elmar Tophoven.

En ce qui concerne les séminaires de traduction du français, Elmar Tophoven en est déjà à son cinquième, si l'on ne compte pas les séminaires réalisés au cours des années 70 au Goethe Institut de Paris, des séminaires entre spécialistes et en bonne intelligence, placés sous l'égide d'une faute de traduction : quelqu'un avait traduit le terme allemand de Vereinsmeierei (propension allemande à créer des associations sur tout et n'importe quoi) en laiterie coopérative. Les réunions se sont donc appelées de cette façon. $S^{\prime}$ ajoutent tous les cours prodigués depuis les années 60 dans le cadre de l'ENS, et qu'Elmar Tophoven ne pratiquait pas comme un détenteur de la 
science infuse. Ces cours étaient une forme de traduction collective avec les meilleurs «forts en thème » de France, plus quelques « amateurs éclairés ». A telle enseigne, dans cette turne précédemment occupée par Paul Celan, Elmar Tophoven avait confectionné des abat-jours pour chaque étudiant, avec des bouteilles en verre de jus d'orange, remplies de cailloux. La turne tout à fait glauque en devenait un lieu non seulement plus attrayant, mais surtout égalitaire. Enfin, depuis la fin des années 70, Elmar Tophoven intervient dans le cadre des Rencontres d'Esslingen qui sont organisées dans le cadre du syndicat des traducteurs littéraires. Il est la cheville ouvrière des séances de travail des traducteurs du français vers l'allemand. Si l'on ajoute la façon dont il a traduit de nombreux textes depuis la fin des années 50 avec son épouse, en étroite collaboration avec Samuel Beckett, sans oublier le travail important des lecteurs de maisons d'édition, Elmar Tophoven est quelqu'un qui adore traduire avec les autres, mais pas pour briller. Ce qui compte, pour lui, c'est la bonne solution inscrite nulle part, qui va permettre d'avancer.

Malheureusement, dans le cadre du Collège, ces séminaires de formation continue s'inscrivent dans des démarches qui n'ont rien à voir avec son art de la traduction conviviale. Qu'on imagine un peu : les professionnels doivent payer de leur personne en traduisant un chapitre de L'épreuve de l'étranger, puis venir assister à Straelen à ce séminaire. Beaucoup de travail sans raison valable, sinon des considérations de gestion des fonds octroyés alors que le but du jeu était, depuis le début, de permettre à des traducteurs de venir à Straelen sans payer, pour y trouver un milieu de traduction optimal. Depuis 35 ans, un certain nombre de traducteurs ont pu en jouir, mais ces séminaires, de plus en plus alignés sur l'enseignement universitaire, n'ont rien à voir avec l'esprit du lieu.

Les participants doivent traduire chacun un chapitre de l'ouvrage et remettre cette traduction brute à une date donnée avant le séminaire. A plusieurs reprises, ce travail est retardé par la recherche des citations. La perspective d'une publication n'est pas donnée, alors que c'est ce qu'il aurait fallu faire!

On est en présence de toute la faconde culturelle spécifique au domaine de la traduction. Des éditeurs payent des séminaires pour 
améliorer le travail de leurs soutiers, mais ils demandent du temps et des efforts. Au lieu de cela, un éditeur aurait dû profiter de ces séminaires pour mettre en route la parution de L'épreuve. Cette parution aurait payé les efforts des acteurs, et elle aurait pu agir en multipliant le nombre de traducteurs sensibilisés à l'œuvre d'Antoine Berman.

Mon expérience passée dans une famille de traducteurs m'a bien montré, au-delà de ceci, à quel point les traducteurs sont méprisés par les éditeurs, d'une manière générale. Il n'est donc pas étonnant que les éditeurs n'ont pas forcément envie de publier Antoine Berman. Simplement, ce genre de considérations n'est pas partagé à l'époque par le Syndicat des traducteurs. Le Collège est depuis 1980 quelque chose comme une maison d'écrivain de l'Europe de l'Est.

Ce projet de traduction de L'épreuve de l'étranger a eu lieu en plein conflit entre Elmar et son homologue Klaus Birkenhauer qui dirige le Collège. Ce qui contribue sans doute à ce que Birkenhauer déprécie l'ouvrage auprès de la fondation Bosch qui finance le séminaire. La pensée d'Antoine est une plante fragile à l'époque, il est facile de minimiser la portée d'un ouvrage français qui se rapporte à la pensée allemande, avec l'argument que ce n'est qu'une thèse.

Antoine se rend à Straelen pour quelques jours et assiste aux travaux qui consiste chaque jour à reprendre un chapitre de façon collective. Il n'a pas vraiment idée de là où il met les pieds. Par contre, je suppose que le choix de traduire L'épreuve, de la part de mon père, avait aussi comme but de faire venir Antoine Berman à Straelen.

\section{Refaire le monde}

Peut-on imaginer de traduire collectivement L'épreuve? C'est tout de même une bonne question à poser dans ce monde, puisque la démarche doit être aussi une plongée commune dans le texte. Mais il n'aurait pas fallu le faire comme cela. L'épreuve a ceci de particulier qu'elle commence par une sorte de préface qui est en fait un manifeste, "La traduction au manifeste", rédigée en 1981, il y a donc quarante ans. Après cette thèse, Antoine Berman prend du recul et rédige de fait un manifeste, mais ce n'est pas le seul de ses 
écrits. « La traduction et ses discours » est aussi un manifeste, et l'Amyot idem.

Il fallait se concentrer sur ce texte, bien le comprendre avec Antoine Berman présent, en déterminer une stratégie de traduction et puis, sur cette base, travailler le reste puis se retrouver pour un lissage final. C'est ce qu'il faudrait refaire maintenant s'il y avait un fond pour traduire les textes essentiels et les faire paraître en ligne indépendamment des éditeurs.

J'ai retraduit le manifeste car j'avais peur que la traduction se casse les dents sur ce qui est encore, dans la prose d'Antoine Berman, tributaire des usages d'écriture des sciences humaines. Après la traduction, j'ai commencé à regarder la traduction de mon père, très méticuleuse, et c'est au mieux un échange, sinon plutôt que c'est moi qui apprends. "Le Manifeste » du début de L'épreuve est ici, dans les archives Tophoven rapatriées à Straelen, très bien traduit et inédit, voilà à peu près la situation culturelle désastreuse entre l'Allemagne et la France ces dernières décennies. Une grande partie des choses importantes écrites d'un côté ou de l'autre ne sont pas traduites, pas publiées. La préférence allemande va vers des philosophies françaises loufoques, quant à la France, la désaffection qui y a cours par rapport à la pensée allemande est ahurissante.

Grâce au travail de Solange Arber, à la réunion des archives sur un même toit dans la maison familiale Tophoven à Straelen, puis à la possibilité de parler de la relation entre Berman et Tophoven, ce manifeste ne retournera pas dans sa boîte. Avec Isabelle Berman, nous allons nous employer à ce qu'il soit visible en Allemagne, Suisse, Autriche, 40 ans après sa rédaction. Et avec un peu de chance, on continuera par les autres chapitres de L'épreuve, puis par les autres œuvres d'Antoine Berman.

\section{Eléments autobiographiques optionnels}

Je connais Isabelle et Antoine par mes parents. Il a toujours été beaucoup question de traduction à la maison. Mes parents m'ont élevé comme un enfant bilingue. Après hésitations, je me suis engagé provisoirement dans la voie d'études germaniques, sans doute par manque d'imagination, et pour atteindre vite une indépendance financière. 
Je ne prends pas part au séminaire de Straelen car je suis en train de préparer l'agrégation, mais mon amie et future épouse, qui a rédigé son mémoire de maîtrise sur l'analyse des fiches de traduction de Mercier et Camier, y participe. Je me souviens simplement qu'Antoine m'a dit ensuite que la seule traduction correcte était la sienne.

Après le décès de mon père, j'étais en poste comme doctorant à Nantes et après l'étape du DEA en 1988, je repoussais le travail sur ma thèse en me consacrant notamment au théâtre. Mais l'occasion m'avait été donnée d'enseigner la théorie de la traduction. J'y étais gêné d'évoquer le travail de mon père, mais je crois bien que j'ai abondamment puisé dans L'épreuve pour préparer mes cours. A l'époque, au printemps 1990, nous avons organisé à Nantes, ma mère et moi, une sorte de petite exposition autour de la traduction transparente. Comme ma compagne (celle qui avait traduit un chapitre de L'épreuve) vivait à Paris, il m'est arrivé d'y assister à une intervention d'Antoine, et à cette occasion, Isabelle m'a fait part de sa maladie sans qu'à l'époque elle soit considérée comme létale. Ma période de libération après le décès de mon père n'a été que de courte durée et j'ai bientôt plongé dans une profonde déprime. Dans ce contexte, je me suis rapproché d'Antoine pour lui proposer de l'aider au Centre Amyot. Mais Antoine était déjà fort malade, les événements se sont accélérés jusqu'à sa mort. Au lieu de l'aider au Centre, j'ai un peu aidé Isabelle, tout en achevant ma thèse et en commençant déjà une nouvelle vie de journaliste. Ensuite, cette vie professionnelle m'a éloigné d'Isabelle et j'ai assisté de loin à ses efforts infinis pour porter les nombreuses traces de la pensée d'Antoine vers la publication. Cela a conduit à accueillir rue St Jacques une réception à l'occasion de la parution du John Donne en 1995.

J'ai quitté l'enseignement en 1995 pour devenir journaliste. A partir de 2005, je me suis mis à écrire et Isabelle a accompagné cela avec bienveillance, jusqu'à aujourd'hui, et depuis un an, elle est vraiment le témoin de mon travail. Il m'arrive de me traduire, dans les deux sens, et aussi d'écrire des sortes de nouvelles qui recourent aux deux langues en même temps. Il me semblait que l'allemand perdait pour moi en importance, mais à l'occasion de mon long séjour en Allemagne à cause du Covid, cette langue a repris le dessus comme langue de création. J'applique plus ou moins dans 
l'écriture une sorte de transparence dérivée de la méthode de traduction de mon père, pas avec des fiches, mais dans la même perspective de mise à jour de processus. J'ai une prédilection pour les modes de traduction hétérodoxes comme les traductions signifiantes à la Brinkmann ou d'autres modes, sachant que l'auto-traduction est aussi une forme hétérodoxe. Ma seule publication est un texte que mon ami Max de Carvalho m'a attribué sur la base de son propre travail à partir de mes mails, une parution qui remonte à plus de dix ans dans le dernier exemplaire de sa revue La Treizième et qui n'a pas grand-chose à voir avec ce que j'écris. J'ai traduit il y a quelques années un recueil de Max de Carvalho, à sa demande et du français en allemand, mais le projet n'a pas abouti à une publication. De même, j'ai traduit sans publication un ouvrage de ma mère de l'allemand en français.

N'étant plus universitaire, et ne rentrant pas dans le cadre traditionnel de la traduction, je n'en reste pas moins fasciné par la pensée d'Antoine. Je partage tout à fait, dans la pratique, son approche pulsionnelle et érotique de la traduction. Sauf que cela se passe chez moi ailleurs, sans la censure éditoriale, sans partage, sans critique. Non que je refuse de partager, mais ce travail jusqu'ici ne mène pas à des publications, sans doute parce qu'il n'est pas assez bon, ou pas encore assez bon. Cela est également une conséquence de l'échec d'une reconversion tentée à partir de 2005 et jusqu'en 2007, vers le métier d'écrivain. J'ai accepté d'écrire ce texte à cause de la soutenance de la thèse de Solange Arber sur la traduction transparente. Je suis bloqué depuis de longs mois en Allemagne mais quelques ouvrages d'Antoine sont disponibles ici dans les archives familiales, ainsi que toute la documentation relative au projet de traduction de L'épreuve.

Cet article, malgré le travail de traduction du « Manifeste », est venu après un travail que je n'ai pas pu faire passer après, un texte en allemand sur ma famille, traduit à peut près en même temps en français, où il s'appelle: Plaisir originel.

\section{Conclusion}

Par le destin, le temps a manqué pour qu'une convergence entre la pensée d'Antoine et la pensée de mon père ne s'opèrent. L'analyse qu'Antoine fait 
du travail d'Amyot, il pourrait la faire du travail de mon père avec ses milliers de fiches, sa démarche d'approche de la traduction proprement dite, son effort de synthèse, sa transmission, les échanges. Mais tout cela est resté en suspens depuis 35 ans. A l'inverse, la pensée d'Antoine aurait enrichi l'approche de mon père, par exemple par la notion de stratégie de traduction, qui, au moment où Antoine l'a formulée, était parfaitement hérétique car le monde culturel ne supportait pas l'idée d'un arbitraire de la traduction. Jusqu'à maintenant prévaut une idée reçue d'une sorte de norme de traduction latente. Une absence de recul que partageait d'ailleurs mon père.

Mon père aurait pris un immense plaisir à lire l'Amyot, pas seulement à cause de la flatterie finale. Alors qu'il scrutait désespérément la linguistique à la recherche d'outils complémentaires pour sa méthode, en lisant tout ce qui lui passait sous la main, il s'intéressait aussi à tout ce qui paraissait en rapport avec la traduction et son histoire. Si la vie leur en avait laissé le temps, non seulement ils se seraient retrouvés, mais cet âge de la traduction se serait prolongé. Mais aujourd'hui, les Collèges existent, les archives Tophoven, les ouvrages d'Antoine. Tout cela a une valeur inestimable. Il est regrettable que nous vivions à une époque où les migrations sont perçues, au moins jusqu'ici, comme une menace et pas comme l'opportunité d'une rencontre culturelle profonde. Que les Collèges ne soient pas devenus en 2015 un lieu d'hébergement des traducteurs fuyant la Syrie. Que l'heure est au réveil ignoble du nationalisme et des démons. Il n'y a pas de quoi être fier de la période culturelle que nous vivons en Europe. Mais la page va peut-être se tourner et ouvrir un nouveau chapitre où la pensée d'Antoine figurera en bonne place, ainsi que la pratique collaborative et collective prônée par mon père.

\section{Bibliographie}

ARBER, Solange. Elmar Tophoven et la traduction transparente. Thèse présentée à Sorbonne Université et Université de Lausanne le 12 décembre 2012.

BERMAN, Antoine. L'épreuve de l'étranger: Culture et traduction dans l'Allemagne romantique. Éditions Gallimard, 1984. 
BERMAN, Antoine. La traduction et ses discours. Confrontation, n. 16, automne 1986.

BERMAN, Antoine. In Memoriam Elmar Tophoven. TTR, 2(1), p. 9, 1989.

BERMAN, Antoine. Pour une critique des traductions : John Donne. Paris : Gallimard, 1995.

BERMAN, Antoine. Jacques Amyot, traducteur français : Essai sur les origines de la traduction en France. Paris : Belin, 2012.

KUHN, Irène. Antoine Bermans Entwurf einer "produktiven" Übersetzungskritik. (Transfer, 15) Narr, Tübingen 2007.

PÜTTER, Rolf. Aus der Erfahrung des Denkens. Rezensionszeitschrift zur Literaturübersetzung, 2010. URL: http://www.relue-online.de/2010/07/ausder-erfahrung-das-denken/

\section{Résumé}

A la fin de la dernière œuvre parue d'Antoine Berman, son ami Elmar Tophoven est mentionné comme un traducteur ayant la copia qui caractérise le travail de Jacques Amyot. Cette mention fait suite à la notice nécrologique publiée par Antoine Berman après la mort du traducteur. Pour autant, malgré leur amitié, les trajectoires et la pensée des deux penseurs de la traduction ne se sont croisées qu'imparfaitement.

Mots clés : Antoine Berman ; Elmar Tophoven ; Traduction transparente ; L'Épreuve de l'étranger ; Allemagne ; Solange Arber.

\section{Resumo}

No final da última obra publicada de Antoine Berman, seu amigo Elmar Tophoven é mencionado como um tradutor que tem a copia, que caracteriza o trabalho de Jacques Amyot. Essa menção dá seguimento ao obituário que Berman publicou após a morte do tradutor alemão. No entanto, apesar da amizade, as trajetórias e a reflexão dos dois pensadores da tradução se cruzam apenas imperfeitamente.

Palavras-chave: Antoine Berman; Elmar Tophoven; Tradução transparente; A prova do estrangeiro; Alemanha; Solange Arber. 